\title{
Relativistic Equations of Motion Based on Equality of Heavy and Inertial Mass
}

\author{
N. Perković, M. Stojić
}

\begin{abstract}
The paper presents the dependence on heavy masses and its position in the central symmetric static field. On the basis of the equivalence of the heavy and inertial masses, the equation of the point object mass $m$ in the mass gravity field of mass $M$ is derived, with $M \gg m$. The account was conducted for observers in an inertial and noninertial system. From the obtained solutions of the equation we calculated the difference between the light defletion and perihelion shift by a short procedure.
\end{abstract}

Index Terms - Relativistic Equations, Inertial Mass.

\section{INTRODUCTION}

Highlight Gravity force is one of the fundamental forces in nature. The term gravitational field is also related to it. The concept of this field is based on the fact that mass changes the space in which it is located meaning that on some other mass there is a force that depends on the strength of the field. The gravitational field has its physical reality that manifests itself at every point of the space, so all bodies regardless of their mass with the same initial conditions are the same in the same way. [1]

In physics we measure two masses, gravitational and inertial. It has been experimentally determined that the ratio of these masses is constant and equal for all types of substances.[2]

The equality of gravity and inertial mass is postulated in Einsten's general theory of relativity it explores new concepts of mass and space and time, so that the dimensions mentioned are only related to one observer.[3]

One such attempt is presented in this paper. A description of the point-of-motion of the mass object in the gravitational field of mass $M$ is presented, with $M \gg m$.

The account was executed for the observer in the inertial and noninertialsystem. Using the basic set of relativistic mechanics, equations of motion in the gravitational field were obtained.

\section{MOTION IN A CENTRAL-SYMMETRIC GRAVITATIONAL FIELD}

We will consider the motion of a point body $m$ in a gravitational field, taking into account the relativistic change of mass towards Einsten's $E=m c^{2}$. Suppose that the dotted body masses in the gravitational field of heavy mass $M$ with $M>m$.

Gravity field of heavy mass $M$ is:

N. Perković , Rudjer Bošković Institute, Zagreb, Croatia

M. Stojić, University North, Varazdin, Croatia
$\vec{g}=-\frac{G M}{|\vec{r}|^{3}} \vec{r}(1)$

where it is:

$\vec{r}=\vec{r}_{0}|\vec{r}|$

Introducing gravity radius:

$R=G \frac{M}{c^{2}}$

The gravitational field will be:

$\vec{g}=-\frac{R c^{2}}{|\vec{r}|^{3}} \vec{r}(3)$

The gravitational force between the two bodies will be:

$\vec{F}=-m \vec{g}=-\frac{R c^{2} m}{|\vec{r}|^{3}} \vec{r}(4)$

The work of this force in the gravitational field source of mass $\mathrm{M}$ will be:

$$
d W=\vec{F} d \vec{r}=-\frac{R c^{2} m}{|\vec{r}|^{2}} d \vec{r}
$$

Since it is:

$d E_{k}=-d U=c^{2} d m(6)$

we can equalize equations (5) and (6) so we obtain a differential equation of the form:

[1] $\frac{d m}{m}=-\frac{R}{|\vec{r}|^{2}} d|\vec{r}|(7)$

By inegrating the left and right sides of the equation (7) we get that:

$m=m_{\infty} e^{\frac{R}{|\vec{r}|}}$

Where $m=m_{\infty}$ when $\overrightarrow{|r|} \rightarrow \infty$

Equation (8) shows dependence of the point of the dot body in the gravitational field of the spring mas $\mathrm{M}$.

In the infinitesimal environment of the dotted body mass $\mathrm{m}$ the velocity of motion can be considered constant so the mass $\mathrm{m}$ can be expressed by the relativistic equation

[2] $m=\frac{m_{0}}{\sqrt{1-\frac{1}{c^{2}}\left(\frac{d \vec{r}}{d T}\right)^{2}}}$

Where in: 
$\vec{v}=\frac{d \vec{r}}{d T}=\vec{\imath}_{\alpha} \frac{d x^{\alpha}}{d T}(10)$

where are the $\quad x^{\alpha}$ and $x^{0}=c T$

coordinate of the rectangular system where T own time of the imaginary observer is located at the origin of this system.Assuming that the masses in equation (8) and (9) are equal, then we get a new relation:

$$
m e^{\frac{R}{|\vec{r}|}}=\frac{m_{0}}{\sqrt{1-\frac{1}{c^{2}}\left(\frac{d \vec{r}}{d T}\right)^{2}}}(11)
$$

Using polar coordinates and quadratic equations we have:

$$
\left(\frac{m_{0}}{m_{\infty}}\right)^{2}=e^{\frac{2 R}{|\vec{r}|}}\left(1-\frac{|\vec{r}|^{2}+|\vec{r}|^{2} \dot{\varphi}^{2}}{c^{2}}\right)(12)
$$

If we derive this equation by observer time $\mathrm{T}$, we get:

$$
-\frac{R c^{2}}{|\vec{r}|^{2}}+\frac{R}{|\vec{r}|^{2}}|\dot{\vec{r}}|^{2}+R \dot{\varphi}^{2}-|\ddot{\vec{r}}|-|\vec{r}| \dot{\varphi}^{2}-|\vec{r}|^{2} \dot{\varphi} \frac{d \varphi}{d|\vec{r}|}=
$$

Using another Neton's law in relativistic form, we have:

$$
\vec{F}=\frac{\vec{d} P}{d T}=\frac{d m}{d T} \frac{\vec{d} r}{d T}+m \frac{d^{2} \vec{r}}{d T^{2}}
$$

Since the force of the central F can be written:

$$
\begin{aligned}
& \quad \vec{F}=\left[m|\ddot{\vec{r}}|-|\vec{r}| \dot{\varphi}^{2}+\frac{d m}{d T}|\dot{\vec{r}}|\right] \vec{r}_{0}+[m|\dot{\vec{r}}| \dot{\varphi}+ \\
& m d d \operatorname{Tr} \varphi+d m d \operatorname{Tr} \varphi \Phi O(15)
\end{aligned}
$$

The second part of the equation with the vector $\overrightarrow{\Phi_{0}}$ will be zero so we get a new equation

$$
\frac{\dot{m}}{m}+2 \frac{|\dot{\vec{r}}|}{r}+\frac{\ddot{\varphi}}{\dot{\varphi}}=0(16)
$$

we can write in the form of:

$\frac{d}{d T} \ln \left(m|\dot{\vec{r}}|^{2} \dot{\varphi}\right)=0(17)$

From theequation (17) it follows:

$$
p_{\varphi}=m|\dot{\vec{r}}|^{2} \dot{\varphi}=\text { const. } .(18)
$$

which means that when the angular quantity is dispensed by the mass replacement $m$ of equation 8 in equation 18 , we obtain:

$$
\frac{d \dot{\varphi}}{d|\vec{r}|}=-\frac{2 \dot{\varphi}}{|\vec{r}|}\left(1-\frac{R}{2|\vec{r}|}\right)
$$

so the equation (13) becomes:

$$
\begin{aligned}
& -\frac{R c^{2}}{|\vec{r}|^{2}}+\frac{R \dot{\vec{r}}^{2}}{|\vec{r}|^{2}}-|\ddot{\vec{r}}|+|\vec{r}| \dot{\varphi}^{2}=0(20) \\
& |\vec{r}|=\frac{1}{\rho}
\end{aligned}
$$

Using the substitution, we are finally gaining the motion for the imaginative observer's center of the rectangular coordinate system set in the dot body mass $\mathrm{m}$ at moment $\mathrm{T}=$ 0

$$
\ddot{\rho}+\rho=p^{-1}+R\left(\rho^{2}+\dot{\rho}^{2}\right)
$$

Where it is:

$$
p^{-1}=\frac{R c^{2} m_{0}^{2}}{p \varphi^{2}}
$$

While:

$$
m^{2}=\frac{m_{0}^{2}}{1-\frac{p_{\varphi}}{m^{2} c^{2}}\left(\rho^{2}+\dot{\rho}^{2}\right)}
$$

Applying the solution to the equation is carried out by the iteration method.In the first iteration the solution reads:

$$
\rho(\varphi)=p^{-1}(1+e \cos \varphi)+R p^{-2}\left(1+e^{2}\right)+R p^{-2} \cdot\left(\varphi \sin \varphi+\frac{1}{2} \cos \varphi\right)
$$

$$
\begin{aligned}
& \quad \rho=0, \varphi=\frac{\pi}{2}+\Delta x \quad \text { arises light deflection } \\
& \text { Za } x=\frac{2 R}{r_{0}}=1.75^{\prime \prime}
\end{aligned}
$$

Where:

$$
e=p \rho(0)-1
$$

The first part of the solution (24) :

$$
\rho(\varphi)=p^{-1}(1+e \cos \varphi)
$$

is the solution of nerelativistic classical equations:

$$
\ddot{\rho}+\rho=p^{-1}
$$

We will now describe the motion of the dot body mass $m$ in gravity field mass $M$ from the neinertial system. The neinertial system is related to our thought-provoking observer, who frees himself in the mass gravity field of mass $M$ (Einstein's lift).

For the description of the motion, we introduce new coordinates $q^{\alpha} ; q^{0}=c t$ lying on the curved space - time hyperbole, where $t$ is the time in the neinertial system, and $c$ the velocity of the light.

In this case the distance between the source of the gravity field and the dotted body will be $\mathrm{r}$ as opposed to $|\vec{r}|$ measuring the starter from the initial system (flat space)

Therefore it will be:

$d \vec{r}=\vec{e}_{\alpha} d q^{\alpha}$ 
and

$$
d \vec{r}^{2}=\vec{e}_{\alpha} \vec{e}_{\beta} d q^{\alpha} d q^{\beta}=g_{\alpha \beta} d q^{\alpha} d q^{\beta}
$$

Where is:

$$
g_{\alpha \beta}=\vec{e}_{\alpha} \vec{e}_{\beta}
$$

We must say that $M>m$ is a gravitational radius: $R=\frac{G M}{c^{2}}$

This field is static, central - symmetric and isotropic so it will be:

$$
g_{0 \alpha}=\frac{\partial x^{0}}{\partial q^{\alpha}}=0, \alpha=1,2, \quad \Theta \text { and } \quad \Theta=\frac{\pi}{2}
$$

Metric tensor is a diagonal matrix with components [4]

$$
\begin{aligned}
& g_{00}=-e^{\frac{-2 R}{r}} \\
& g_{11}=e^{\frac{2 R}{r}} \\
& g_{22}=r^{2} e^{\frac{2 R}{r}}
\end{aligned}
$$

Equation (11) in this system becomes:

$\frac{m_{0}{ }^{2}}{m_{\infty}^{2}}=e^{\frac{2 R}{r}}\left(1-\frac{g_{\alpha \beta} d q^{\alpha} d q^{\beta}}{c^{2} g_{00} d t^{2}}\right)(30)$

Respectively:

$$
\frac{m_{0}^{2}}{m_{\infty}^{2}}=e^{\frac{2 R}{r}}\left(1-e^{\frac{4 R}{r}}\left(\frac{\dot{r}^{2}+r^{2} \dot{\varphi}^{2}}{c^{2}}\right)(31)\right.
$$

Deriving the equation (30) by intermittent observers is obtained:

$$
\rho(\varphi)=p^{-1}(1+e \cos \varphi)+R p^{-2}\left(5+e^{2}\right)+3 R p^{-2} e \cdot\left(\varphi \sin \varphi+\frac{1}{2} \cos \varphi\right)
$$

From the obtained solutions 24 and 25, gravitational deflection of light can be counted, which is caused by massive space objects as well as perihelion motion. [5]

From :

$$
\rho(\varphi)=p^{-1}\left(1+e \cos \varphi\left(1-3 R p^{-1}\right)+\frac{3}{2} R p^{-2} e \cos \varphi\right)
$$

we get:

$$
T=\frac{2 \pi}{1-3 R p^{-1}}=T_{0}+\Delta T \text { and } \Delta T=6 \pi R p^{-1}
$$

which for Mercury is 42 "in 100 years.

\section{CONCLUSION}

By applying the principle of mass and energy relativity, according to Einstein's $E=m c^{2}$, a new relation between the gravitational mass and the field source was derived. The obtained equation (8) shows the mass dependence of its position relative to the source of the field. When this mass equates to the congruent mass (9), using the principle of equivalence of gravitational and inecious mass, a new equation can be defined by the observer's time.
In this way, with the application of the second Netwon Law in the relativistic form, the observation motion equations were obtained in the inertial and noninertial system. Observation the solutions of these equations was carried out by the iteration method by applying a solution to the gravitational deflection of light.

\section{REFERENCES}

[1] L. D. Landau and E. M. Lifshitz, The Classical Theory of Fields, Pergamon Press, 1971.

[2] A. Einstein, How I Constructed the Theory of Relativity, translated by Masahiro Morikawa from the text recorded in Japanese by Jun Ishiwara, Association of Asia Pacific Physical Societies (AAPPS) 
Bulletin, Vol. 15, No. 2, pp. 17-19, April 2005. Einstein recalls events of 1907 in a talk in Japan on 14 December 1922.

[3] A. Einstein, The Meaning of Relativity, Princeton University Press, 2005.

[4] M. Martinis, N. Perković, Relativistic Modification of Newtonian Gravity Law, International Journal of New Technology and Research, ISSN:2454-411,Volume, Issue-2, February 2018, Page 42-46.

[5] Mladen Martinis, Nediljko Perković, Is exponential metric a natural space-time metric of Newtonian gravity?, arXiv:1009.6017, Subjects: General Relativity and Quantum Cosmology (gr-qc). 